\title{
A NOTE ON THE SERUM PROTEINS IN NORMAL INFANTS AND CHILDREN*
}

BY

J. BASIL RENNIE, M.D., F.R.F.P.S.

(From the Department of Paediatrics, Glasgow University, and the Biochemical Laboratory, Royal Hospital for Sick Children, Glasgow.)

As a result of the work of Starling ${ }^{1}$ the important part played by the serum proteins in the pathogenesis of oedema has secured recognition. More recently it has been demonstrated that decrease in serum protein is responsible for a reduction in the calcium content of the blood; the reason for this is that a portion of the calcium exists in combination with protein.

In the literature numerous figures are available for the serum protein content of the blood in normal adults but not for children. With regard to values given for normal children, in most instances, the total protein level, as estimated by the refractometer, is all that is available. The object of this communication is to report the results obtained in twenty-four apparently normal children between two and eleven years and in twenty-two infants and young children under two years by the micro-Kjeldhal method of Howe $^{2}$ as modified by Hawk and Bergheim ${ }^{3}$.

\section{Methods available.}

Before recording the results a brief discussion on the relative merits of the four principal methods of measurement of the serum proteins may be made.

1. The graximetric method. While, according to Peters and van Slyke $^{4}$, this is the most accurate procedure, it involves the use of a large quantity of serum or plasma and for that reason it is unsuitable for investigating the blood of children.

2. The micro-Kjeldhal method. By combustion with acid the protein of the serum or plasma is converted into ammonium sulphate and the amount of nitrogen present is estimated by nesslerization or distillation: albumin and globulin are determined separately by Howe's technique. The procedure is accurate and smaller amounts of blood can be used than in the gravimetric method.

3. The colorimetric method of $\mathbf{W} \mathbf{u}^{5}$. This test is really an estimation of the tyrosine which forms part of the protein molecule. It has been shown by Tachmann and Sobotka ${ }^{6}$ that, while in normal cases results comparable with the Kjeldhal method are obtained, in nephrosis and nephritis the

* The work was done during the tenure of a Carnegie Research Scholarship. 
percentage of serum albumin estimated by this method is too high because the relative percentage of tyrosine in serum albumin and globulin is altered in these conditions.

4. Refractometry. While this procedure for estimating total proteins is much the simplest, there is a general consensus of opinion that the results are too high owing to the presence in the serum of substances other than protein causing refraction of the light rays. Linder ${ }^{7}$ and his co-workers found the total protein of the serum to be $1.59 \mathrm{gm}$. per cent. higher than by the Kjeldhal method in normal subjects. This discrepancy is in the main due to the presence of fat. Kumpf ${ }^{8}$, using Robertson's ${ }^{9}$ refractometric technique, found in normal subjects fair correlation for albumin, globulin and total protein with the Kjeldhal method, but in the lipaemic sera of nephrotic patients total protein and globulin by the refractometer were much too high.

From these remarks it may be concluded that the method of choice is the micro-Kjeldhal.

The serum proteins in older children.

The ages of cases investigated were between two-and-a-half and eleven years. The series consisted of patients convalescent from rheumatism or suffering from enuresis or epilepsy. They were all afebrile and well nourished at the time of the investigation. Table 1 shows the results obtained for the total protein and the albumin and globulin of the serum. Oncotic pressure was calculated from the formula of Govaerts ${ }^{10}$.

Total protein ranged from 6.83 to 8.24 , albumin from 4.45 to 5.94 and globulin from 1.52 to $2.94 \mathrm{gm}$. per cent. The average figures were total protein $7 \cdot 42$, albumin $5 \cdot 15$ and globulin $2 \cdot 27$ gm. per cent. Calculated serum oncotic pressure ranged from 27.8 to $35.5 \mathrm{~mm}$. $\mathrm{Hg}$. These results are comparable with those published in a recent article by Peters and Eisenman ${ }^{11}$ who made one hundred and nine estimations of total serum protein by a modification of Howe's method in fifty-two adults. They found that it lay between 6.3 and $7 \cdot 7 \mathrm{gm}$. per cent. in 90 per cent. of instances. Fifty estimations of serum albumin in thirty-four subjects lay between $4 \cdot 1$ and $6 \cdot 1 \mathrm{gm}$. per cent. but in only one case was the level above $5.5 \mathrm{gm}$. per cent. Serum globulin ranged from 0.9 to $3 \mathrm{gm}$. per cent. In only one instance did it fall below 1.4 gm. per cent. They conclude that the normal limits of variation are:-serum total protein $6 \cdot 0-8.0 \mathrm{gm}$. per cent., serum albumin 4.0-5.5 gm. per cent. and serum globulin 1.4-3.0 gm. per cent.

The results of other workers who have employed Howe's method are given in table 2 together with those obtained in the present series. For purposes of comparison figures obtained by Dr. Jessie W. Ogilvie (personal communication) may be quoted. In forty-five convalescent rheumatic patients total serum protein estimated by the refractometer averaged $8.34 \mathrm{gm}$. per cent. or $0.92 \mathrm{gm}$. per cent. more than the figure obtained by the micro-Kjeldhal method in the present series.

Earlier reports have indicated that globulin is slightly higher in females than in males. In the large series of Peters and Eisenman no difference was observed, a finding in keeping with the results obtained in this work. 
TABLE 1.

THE SERUM PROTEINS BETWEEN THE AGES OF TWO-AND-A-HALF AND ELEVEN YEARS IN NORMAL SUBJECTS.

\begin{tabular}{|c|c|c|c|c|c|}
\hline SEX & $\begin{array}{l}\text { AGE IN } \\
\text { YEARS }\end{array}$ & $\begin{array}{c}\text { TOTAL } \\
\text { PROTEIN : GM. } \\
\text { PER CENT. } \\
\end{array}$ & $\begin{array}{l}\text { Albumin : Gm. } \\
\text { Per Cent. }\end{array}$ & $\begin{array}{l}\text { GLOBULIN : GM. } \\
\text { PER CENT. }\end{array}$ & $\begin{array}{c}\text { Calculated } \\
\text { ONCOTIC PRESSURE } \\
\text { MM. HG. }\end{array}$ \\
\hline $\mathbf{F}$ & 4 & $6 \cdot 84$ & $5 \cdot 32$ & $1 \cdot 52$ & $31 \cdot 4$ \\
\hline $\mathbf{F}$ & $2 \frac{1}{2}$ & $6 \cdot 87$ & $4 \cdot 49$ & $\mathbf{2} \cdot 38$ & $28 \cdot 0$ \\
\hline $\mathbf{M}$ & 5 & $7 \cdot 75$ & $4 \cdot 81$ & $2 \cdot 94$ & $30 \cdot 6$ \\
\hline $\mathbf{M}$ & 5 & $6 \cdot 84$ & $4 \cdot 74$ & $2 \cdot 10$ & 28.9 \\
\hline $\mathbf{M}$ & 5 & $8 \cdot 01$ & $5 \cdot 22$ & $2 \cdot 79$ & $32 \cdot 6$ \\
\hline M & 5 & $8 \cdot 24$ & $5 \cdot 43$ & $2 \cdot 81$ & 33.9 \\
\hline $\mathbf{F}$ & 5 & $7 \cdot 25$ & $4 \cdot 97$ & $2 \cdot 28$ & 30.5 \\
\hline $\mathbf{M}$ & 7 & $7 \cdot 38$ & $5 \cdot 10$ & $2 \cdot 28$ & $31 \cdot 2$ \\
\hline $\mathbf{M}$ & 7 & $7 \cdot 54$ & $5 \cdot 18$ & $2 \cdot 36$ & $31 \cdot 8$ \\
\hline $\mathbf{M}$ & 8 & $7 \cdot 22$ & $4 \cdot 72$ & $2 \cdot 50$ & $29 \cdot 4$ \\
\hline $\mathbf{F}$ & 8 & $6 \cdot 84$ & $4 \cdot 45$ & $2 \cdot 39$ & $27 \cdot 8$ \\
\hline $\mathbf{F}$ & 9 & $7 \cdot 58$ & $5 \cdot 94$ & $1 \cdot 64$ & $34 \cdot 9$ \\
\hline $\mathbf{F}$ & 9 & $7 \cdot 43$ & $4 \cdot 70$ & $2 \cdot 73$ & $29 \cdot 6$ \\
\hline $\mathbf{M}$ & 10 & $7 \cdot 10$ & $4 \cdot 72$ & $\mathbf{2} \cdot 38$ & $28 \cdot 4$ \\
\hline $\mathbf{F}$ & 10 & $7 \cdot 64$ & $5 \cdot 28$ & $2 \cdot 36$ & $\mathbf{3 2} \cdot \mathbf{3}$ \\
\hline $\mathbf{F}$ & 11 & $7 \cdot 78$ & $5 \cdot 27$ & $2 \cdot 51$ & $32 \cdot 9$ \\
\hline $\mathbf{F}$ & 11 & $7 \cdot 27$ & $5 \cdot 52$ & $1 \cdot 75$ & $32 \cdot 8$ \\
\hline $\mathbf{F}$ & 11 & $7 \cdot \mathbf{3 4}$ & $5 \cdot 12$ & $2 \cdot 2 \cdot 2$ & $31 \cdot 2$ \\
\hline $\mathbf{F}$ & 11 & $6 \cdot 83$ & $4 \cdot 99$ & $1 \cdot 84$ & $30 \cdot 0$ \\
\hline $\mathbf{M}$ & 10 & $8 \cdot 02$ & 5.92 & $2 \cdot 10$ & $\mathbf{3 5} \cdot 5$ \\
\hline $\mathbf{M}$ & 8 & $7 \cdot 78$ & $5 \cdot 62$ & $2 \cdot 16$ & $34 \cdot 0$ \\
\hline $\mathbf{F}$ & 9 & $8 \cdot 04$ & $5 \cdot 25$ & $2 \cdot 79$ & $32 \cdot 8$ \\
\hline $\mathbf{M}$ & 8 & $7 \cdot 03$ & $5 \cdot 42$ & $1 \cdot 61$ & $32 \cdot 5$ \\
\hline \multirow[t]{2}{*}{$\mathbf{M}$} & 10 & $7 \cdot 62$ & $5 \cdot 59$ & $2 \cdot 03$ & $33 \cdot 6$ \\
\hline & Average & $7 \cdot 42$ & $5 \cdot 15$ & $2 \cdot 27$ & $31 \cdot 5$ \\
\hline
\end{tabular}

TABLE 2.

The SERUM PROTEINS OF NORMAL ADULTS AND CHILDREN.

\begin{tabular}{|c|c|c|c|c|c|c|c|c|}
\hline AUTHOR & $\begin{array}{l}\text { SERUM } \\
\text { OR } \\
\text { PLASMA }\end{array}$ & $\begin{array}{c}\text { No. OF } \\
\text { CASES }\end{array}$ & $\mathrm{AGE}$ & SEX & $\begin{array}{l}\text { TOTAL } \\
\text { PROTEIN } \\
\text { GM. } \\
\text { PER CENT. }\end{array}$ & $\begin{array}{l}\text { Albunis } \\
\text { gai. } \\
\text { Per CENT. }\end{array}$ & $\begin{array}{l}\text { GLOBULiN } \\
\text { GM. } \\
\text { PER CENT. }\end{array}$ & $\begin{array}{c}\text { Calculated } \\
\text { ONCOTIC } \\
\text { PRESSURE } \\
\text { MM. HG. }\end{array}$ \\
\hline Present & S & 12 & Childoren & $\mathbf{M}$ & $7 \cdot 54$ & $5 \cdot 20$ & $2 \cdot 34$ & $31 \cdot 9$ \\
\hline $\begin{array}{c}\text { SERIES } \\
\text { McLuRE }\end{array}$ & $\mathbf{S}$ & 12 & , & $\mathbf{F}$ & $7 \cdot 32$ & $5 \cdot 11$ & $2 \cdot 21$ & $31 \cdot 2$ \\
\hline ET ALII ${ }^{12}$ & $\mathbf{P}$ & 3 & , & & $7 \cdot 01$ & $4 \cdot 90$ & $2 \cdot 10$ & $30 \cdot 3$ \\
\hline SALVESEN $1::$ & $\mathbf{P}$ & $\begin{array}{l}16 \\
16\end{array}$ & $\begin{array}{c}\text { Anults } \\
, y\end{array}$ & $\stackrel{M}{F}$ & $\begin{array}{l}7 \cdot 00 \\
7 \cdot 02\end{array}$ & $\begin{array}{l}4 \cdot 44 \\
4 \cdot 35\end{array}$ & $\begin{array}{l}2 \cdot 58 \\
2 \cdot 68\end{array}$ & $\begin{array}{l}28 \cdot 0 \\
27 \cdot 7\end{array}$ \\
\hline $\begin{array}{l}\text { LINDER } \\
\quad \text { ET ALII }{ }^{7}\end{array}$ & $\mathbf{P}$ & 8 & , , & & $6 \cdot 73$ & $4 \cdot 11$ & $2 \cdot 61$ & $26 \cdot 2$ \\
\hline BRUCKMAN & & & & & & & & \\
\hline ET $A L I I^{14}$ & $S$ & $\begin{array}{r}13 \\
8\end{array}$ & ," & $\underset{\mathrm{F}}{\mathrm{M}}$ & $\begin{array}{l}6 \cdot 93 \\
7 \cdot 61\end{array}$ & $\begin{array}{l}5 \cdot 06 \\
4.98\end{array}$ & $\begin{array}{l}1 \cdot 89 \\
2 \cdot 62\end{array}$ & $\begin{array}{l}30 \cdot 5 \\
31 \cdot 0\end{array}$ \\
\hline $\begin{array}{l}\text { KUMPF } 8 \\
\text { MOORE AND }\end{array}$ & $S$ & 8 & ", & & $7 \cdot 17$ & 4.82 & $2 \cdot 34$ & $29 \cdot 8$ \\
\hline $\begin{array}{l}\text { VAN SLYKE }^{15} \\
\text { WIENER AND }\end{array}$ & $\mathbf{P}$ & 9 & ," & & $7 \cdot 10$ & $4 \cdot 30$ & $2 \cdot 80$ & $27 \cdot 7$ \\
\hline WIENER $^{23}$ & S & 20 & , & & $6 \cdot 40$ & $4 \cdot 60$ & $1 \cdot 70$ & $27 \cdot 7$ \\
\hline
\end{tabular}


The serum proteins under two years of age.

Several authors, using the refractometer, have reported that the serum proteins in the first months of life are lower than the adult level. MelioLeitao $^{16}$ and Bakwin and Rivkin ${ }^{17}$ found that the adult level for serum total protein was not attained until eighteen months. Kylin ${ }^{18}$ stated that under two years, serum total protein ranged from 4.6 to $6.5 \mathrm{gm}$. per cent. as compared with $6 \cdot 4$ to $8 \cdot 6 \mathrm{gm}$. per cent. in subjects over two years. $\mathrm{He}$ also measured serum oncotic pressure and found that it ranged from 17.0 to $26.0 \mathrm{~mm}$. $\mathrm{Hg}$. under two years compared to 29.0 to $37.0 \mathrm{~mm}$. $\mathrm{Hg}$. over that age. Levy ${ }^{19}$ estimating total protein in twenty-nine infants, obtained results comparable with those of Kylin. Darrow and Cary ${ }^{20}$ lend support to the results of these previous investigators with the following figures.

\begin{tabular}{|c|c|c|c|c|c|c|}
\hline AGE. & & No. & $\begin{array}{c}\text { TOTAL } \\
\text { PROTEIN } \\
\text { GM. } \\
\text { PER CENT. }\end{array}$ & $\begin{array}{l}\text { ALBUMIIN } \\
\text { GM. } \\
\text { PER CENT. }\end{array}$ & $\begin{array}{l}\text { GLOBULIN } \\
\text { GM. } \\
\text { PER CENT. }\end{array}$ & $\begin{array}{l}\text { Calculated } \\
\text { oncotic } \\
\text { PRESSURE } \\
\text { limi. HG. }\end{array}$ \\
\hline 'Premature' ... & $\cdots$ & 26 & 4.94 & $3 \cdot 58$ & $1 \cdot 18$ & $20 \cdot 4$ \\
\hline NORMaL NEWBORN & $\ldots$ & 20 & $5 \cdot 52$ & $3 \cdot 73$ & 1.78 & $23 \cdot 0$ \\
\hline 5-8 MONTHS & $\ldots$ & 14 & $6 \cdot 29$ & $4 \cdot 28$ & $2 \cdot 01$ & $26 \cdot 3$ \\
\hline
\end{tabular}

The last-mentioned workers and Levy used the Kjeldhal method.

Ray and Phatak ${ }^{21}$ on the other hand employing Greenberg's"2 modification of Howe's method found in twenty new-born infants the following averages:-Total protein $7.0 \mathrm{gm}$. per cent., albumin $5.1 \mathrm{gm}$. per cent. and globulin $1.9 \mathrm{gm}$. per cent. Govaert's formula applied to these figures gives a calculated oncotic pressure of $30.7 \mathrm{~mm}$. $\mathrm{Hg}$.

In this work the serum proteins have been estimated in twenty-two apparently normal infants whose ages ranged from three to twenty-three months. The majority of the cases suffered from rickets or some disease of the nervous system. None was acutely ill or had fever. Table 3 shows the results. Serum total protein ranged from 6.04 to $8.0 \mathrm{gm}$. per cent., albumin from 4.12 to $5.91 \mathrm{gm}$. per cent. and globulin from 1.13 to $2.82 \mathrm{gm}$. per cent. Calculated oncotic pressure ranged from 26.5 to $34 \cdot 4 \mathrm{~mm}$. $\mathrm{Hg}$. The average figures were total protein 7.08 , albumin 4.95 and globulin $2 \cdot 13 \mathrm{gm}$. per cent., and the average calculated oncotic pressure was $30.2 \mathrm{~mm} . \mathrm{Hg}$.

Comparison of the figures for infants over three months and for older children shows little difference either in range or in average. If the infants are grouped in age periods (table 4) a slight rise is seen in serum albumin and a slight decrease in serum globulin with advancing age. The slight rise in oncotic pressure which also occurs reflects the increase in albumin. 
TABLE 3.

NORMAL SERUM PROTEINS UNDER TWO YEARS OF AGE.

\begin{tabular}{|c|c|c|c|c|c|c|c|}
\hline & \multicolumn{3}{|c|}{$A G E$} & $\begin{array}{c}\text { TOTAL } \\
\text { PROTEIN : GM. } \\
\text { PER CENT. }\end{array}$ & $\begin{array}{l}\text { ALBUMIN : GM. } \\
\text { PER CENT. }\end{array}$ & $\begin{array}{l}\text { GLOBULIN : GM. } \\
\text { PER CENT. }\end{array}$ & \multirow{2}{*}{$\begin{array}{c}\text { Calculated } \\
\text { ONCOTIC } \\
\text { PRESSURE } \\
\text { MM. HG. } \\
\\
27 \cdot 8\end{array}$} \\
\hline 15 & WEEKS & $\ldots$ & $\ldots$ & $6 \cdot 87$ & $4 \cdot 49$ & $2 \cdot 38$ & \\
\hline 16 & , & $\ldots$ & $\ldots$ & $7 \cdot 19$ & $5 \cdot 10$ & $2 \cdot 09$ & $30 \cdot 9$ \\
\hline 17 &, & $\ldots$ & $\ldots$ & $7 \cdot 22$ & $4 \cdot 96$ & $2 \cdot 26$ & $30 \cdot 4$ \\
\hline 19 & , & $\ldots$ & $\ldots$ & $7 \cdot 10$ & $4 \cdot 48$ & $2 \cdot 62$ & $28 \cdot 3$ \\
\hline 27 & , , & $\ldots$ & $\ldots$ & 6.56 & $5 \cdot 43$ & $1 \cdot 13$ & $31 \cdot 5$ \\
\hline 9 & MONTHS & $\ldots$ & $\ldots$ & $7 \cdot 63$ & $5 \cdot 38$ & $2 \cdot 25$ & $32 \cdot 7$ \\
\hline 9 & ,, & $\ldots$ & $\ldots$ & 6.94 & $4 \cdot 12$ & $2 \cdot 82$ & 267 \\
\hline 9 & , , & $\ldots$ & $\ldots$ & $7 \cdot 33$ & $4 \cdot 77$ & $2 \cdot 56$ & $29 \cdot 8$ \\
\hline 10 & , & $\ldots$ & $\ldots$ & $6 \cdot 79$ & 4.92 & $1 \cdot 87$ & $29 \cdot 7$ \\
\hline 11 &,, & $\ldots$ & $\ldots$ & $7 \cdot 72$ & 4.95 & $2 \cdot 77$ & $31 \cdot 1$ \\
\hline 11 & , , & $\ldots$ & $\ldots$ & $6 \cdot 82$ & $4 \cdot 14$ & $2 \cdot 68$ & $26 \cdot 5$ \\
\hline 1 & YE.AR & $\ldots$ & $\ldots$ & 6.04 & $4 \cdot 83$ & $1 \cdot 21$ & $28 \cdot 5$ \\
\hline 1 & , & $\ldots$ & $\ldots$ & $6 \cdot 41$ & 4.92 & $1 \cdot 49$ & $29 \cdot 1$ \\
\hline 13 & MONTHS & $\ldots$ & $\ldots$ & $6 \cdot 31$ & 5.05 & $1 \cdot 26$ & $29 \cdot 5$ \\
\hline 14 &, & $\ldots$ & $\ldots$ & $7 \cdot 69$ & $5 \cdot 21$ & $2 \cdot 48$ & $32 \cdot 1$ \\
\hline 16 &, & $\ldots$ & $\ldots$ & $7 \cdot 64$ & 4.95 & $2 \cdot 69$ & $31 \cdot 0$ \\
\hline 17 &, & $\ldots$ & $\ldots$ & $7 \cdot 91$ & $5 \cdot 39$ & $2 \cdot 52$ & $33 \cdot 2$ \\
\hline 17 & , , & $\ldots$ & $\ldots$ & $6 \cdot 38$ & $5 \cdot 04$ & $1 \cdot 34$ & $29 \cdot 6$ \\
\hline 18 &, & $\ldots$ & $\ldots$ & $8 \cdot 00$ & $5 \cdot 22$ & $2 \cdot 78$ & $32 \cdot 6$ \\
\hline 18 & , , & $\ldots$ & $\ldots$ & $6 \cdot 55$ & $4 \cdot 84$ & 1.71 & $29 \cdot 0$ \\
\hline 20 & , & $\ldots$ & $\ldots$ & $7 \cdot 33$ & $4 \cdot 82$ & $2 \cdot 51$ & $30 \cdot 0$ \\
\hline 23 & , & $\ldots$ & $\ldots$ & $7 \cdot 31$ & 5.91 & $1 \cdot 40$ & $34 \cdot 4$ \\
\hline & Ave & RAGE & $\ldots$ & $7 \cdot 08$ & $4 \cdot 95$ & $2 \cdot 13$ & $30 \cdot 2$ \\
\hline
\end{tabular}

TABLE 4 .

The Sert'M proteins in INFANTS accordng to aGe.

\begin{tabular}{|c|c|c|c|c|c|c|}
\hline AGE & & $\begin{array}{l}\text { No. of } \\
\text { CASES }\end{array}$ & $\begin{array}{c}\text { Total } \\
\text { PROTEIN } \\
\text { GMT. } \\
\text { PER CENT. }\end{array}$ & $\begin{array}{l}\text { AlBUMAN } \\
\text { GM. } \\
\text { PER CENT. }\end{array}$ & $\begin{array}{l}\text { GLOBULix } \\
\text { GM. } \\
\text { PER CENT. }\end{array}$ & $\begin{array}{c}\text { Calculatei } \\
\text { oncotic } \\
\text { Pressure } \\
\text { MM. HG. }\end{array}$ \\
\hline is $\ldots$ & $\ldots$ & 4 & $7 \cdot 09$ & 4.75 & $2 \cdot 34$ & $29 \cdot 4$ \\
\hline $\begin{array}{lll}\text { is } & \ldots\end{array}$ & $\ldots$ & 7 & $7 \cdot 11$ & $4 \cdot 81$ & $2 \cdot 30$ & $29 \cdot 8$ \\
\hline$\ldots$ & $\ldots$ & 6 & $7 \cdot 00$ & $5 \cdot 06$ & 1.94 & $30 \cdot 5$ \\
\hline$\ldots$ & $\ldots$ & 5 & $7 \cdot 11$ & $5 \cdot 16$ & 1.95 & $31 \cdot 1$ \\
\hline
\end{tabular}

Summary.

Since few analyses of serum proteins by the micro-Kjeldhal method in normal infants and children have been published, the results of estimations in twenty-four older children and twenty-two infants are reported. No essential difference from the values obtained in adults was found in children varying in age from three months to eleven years. 


\section{REFERENGES.}

1. Starling, E. H., J. Physiol., London, 1895-6, XIX, 312.

2. Howe, P. E., J. Biol. Chem., Baltimore, 1921, XLIX, 109.

3. Hawk, P. B., \& Bergeim, O., Practical Physiological Chemistry, London, 1926.

4. Peters, J. P., \& Van Slyke, D. D., Quantitative Clinical Chemistry, II, London, 1932.

5. Wu, H., J. Biol. Chem., Baltimore, 1922, XLI, 33.

6. Tachmann, I. P., \& Sobotka, H., ibid., 1932, XCVIII, 35.

7. Linder, G. C., et alii, J. Exp. Med., New York, 1924, XXXIX, 887.

8. Kumpf, A. E., Arch. Path., Chicago, 1931, XI, 335.

9. Robertson, T. B., J. Biol. Chem., Baltimore, 1915, XXII, 233.

10. Govaerts, P., Compt. rend. Soc. biol., Paris, 1925, XCIII, 441; ibid., 1926, $\mathrm{XCV}, 724$.

11. Peters, J. P., \& Eisenman, A. J., Am. J. Med. Sc., Philad., 1933, XLXXXVI, 808.

12. McLure, W. B., et alii, Arch. Int. Med., Chicago, 1933, LI, 819.

13. Salvesen, H. A., Acta Med. Scand., Stockholm, 1926-7, LXV, 147.

14. Bruckman, F. S., et alii, J. Clin. Invest., New York, 1930, VIII, 577.

15. Moore, N. S., \& Van Slyke, D. D., loc. cit., 337.

16. Mello-Leitao, Am. J. Dis. Child., Chicago, 1916, XI, 214.

17. Bakwin, H., \& Rivkin, H., ibid., 1924, XXVII, 340.

18. Kylin, E., Acta paediat., Uppsala, 1932, XIV, 160.

19. Levy, E., Réu. franç. de Paediat., Paris, 1928, IV, 596.

20. Darrow, D. C., \& Cary, M. K., J. Pediat., St. Louis, 1933, III, 573.

21. Ray, H. H., \& Phatak, N. M., Am. J. Dis. Child., Chicago, 1930, XL, 549.

22. Greenberg, D. M., J. Biol. Chem., Baltimore, 1929, LXXXII, 545.

2\%. Wiener, H. J., \& Wiener, R. E., Arch. Int. Med., Chicago, 1930, XLVI, 236. 